\section{Center and surgeon procedural volumes affect functional outcome after knee replacement}

High procedural volumes reduce perioperative mortality and complication rates for a range of procedures-which has prompted US qualityimprovement organizations to endorse selective referral to high-volume centers for major cardiac and oncologic procedures. In this study, Katz and colleagues explore whether this relationship extends to health-related quality of life; they investigated whether functional outcome after total knee replacement (TKR) is influenced by the procedural volume of the center and/or surgeon.

The cohort included 932 Medicare patients from four US states who underwent primary TKR (96\% for osteoarthritis). The patients completed a questionnaire that assessed their functional status according to the Western Ontario and McMaster Universities Osteoarthritis Index (1-100 scale; $100=$ best). Two years after surgery, patients operated on in lowvolume centers ( $\leq 25$ TKRs performed annually) by low-volume surgeons (performed $\leq 6$ TKRs annually) were twice as likely to have a poor functional status score (Western Ontario and McMaster Universities Osteoarthritis Index $<60$ ) than patients operated upon in a high-volume environment for both center and surgeon together.

Interestingly, no association was found between pain score (also assessed 2 years postoperatively) and either surgeon or center procedural volume; the authors suggest that this finding is because the cause of pain (the arthritic knee) is always removed by TKR, whereas functional outcome is influenced by many factors including the surgeon's experience of performing this procedure.

Original article Katz JN et al. (2007) Association of hospital and surgeon procedure volume with patient-centered outcomes of total knee replacement in a population-based cohort of patients age 65 years and older. Arthritis Rheum 56: 568-574

\section{Hematopoietic stem cell transplantation is a feasible therapy for refractory vasculitis}

Intensive immunosuppression with cyclophosphamide is the preferred treatment for severe vasculitis, but long-term cyclophosphamide therapy increases patients' risk of secondary malignancies. Immunoablation followed by hematopoietic stem cell transplantation (HSCT) has shown promise in severe autoimmune disease, but data on its use in vasculitic diseases are scarce. Daikeler et al., therefore, performed a retrospective analysis of data from HSCT-treated patients with treatment-refractory vasculitis.

Detailed information on 15 patients (mean age 37 years at HSCT, 6 male) with vasculitis that had not responded to intensive immunosuppression (including cyclophosphamide therapy) at HSCT was obtained from the European League Against Rheumatism and European Bone Marrow Transplantation databases. After immunoablation, 14 patients initially underwent autologous and one patient underwent allogeneic HSCT; three patients required further transplant after relapse, and these subsequent transplants were included in the overall analysis. Overall response to HSCT was $92 \%: 46 \%$ patients had a complete response (off immunosuppression without disease activity), $46 \%$ had a partial response (substantially reduced immunosuppression). The median duration of response at the time of reporting was 45 months. Three patients died (one each from advanced disease, cancer and graft-versus-host disease).

Daikeler and colleagues concluded that HSCT should be considered for patients with preserved organ function who have vasculitis that does not respond to cyclophosphamide, but add that their findings should be confirmed by prospective clinical trials.

\footnotetext{
Original article Daikeler T et al. (2007) Haematopoietic stem cell transplantation for vasculitis including Behçet's disease and polychondritis: a retrospective analysis of patients recorded in the European Bone Marrow Transplantation and European League Against Rheumatism databases and a review of the literature. Ann Rheum Dis 66: 202-207
} 